10,11

\title{
Абсолютная неустойчивость ГЦК-решетки кристаллов инертных газов под давлением
}

\author{
(C) Е.П. Троицкая ${ }^{1}$, Е.А. Пилипенко ${ }^{1, \uparrow}$, E.Е. Горбенко ${ }^{2}$ \\ ${ }^{1}$ Донецкий фозико-технический институт им. А.А. Галкина, \\ Донецк, Украина \\ 2 Луганский национальный университет им. Т. Шевченко, \\ Луганск, Украина \\ ฯ E-mail: pilipenko.katerina@mail.ru
}

(Поступила в Редакцию 25 апреля 2018 г.)

\begin{abstract}
На основе ab initio расчетов фононных частот сжатых кристаллов инертных газов в модели деформируемых и поляризуемых атомов исследуется динамическая нестабильность ГЦК-решетки этих кристаллов. В короткодействующий потенциал, наряду с рассмотренным ранее трехчастичным взаимодействием, связанным с перекрытием электронных оболочек атомов, включены трехчастичные силы, обусловленные взаимной деформацией электронных оболочек соседних атомов. Показано, что учет деформации электронных оболочек атомов дипольного типа в парном и трехчастичном приближениях приводит к размягчению „критических“ колебаний и абсолютной неустойчивости ГЦК-решетки при давлениях больше критических $p>p_{c}$. Для легких кристаллов $\mathrm{Ne}$ и $\mathrm{Ar}$ при сжатиях равных $0.76\left(p_{c}=422 \mathrm{GPa}\right)$ и $0.71\left(p_{c}=405 \mathrm{GPa}\right)$ соответственно размягчение продольной моды наблюдается на границе зоны Бриллюэна в точке $L$, для тяжелых кристаллов $\mathrm{Kr}$ и Хе при сжатиях $0.686\left(p_{c}=240 \mathrm{GPa}\right)$ и $0.605\left(p_{c}=88 \mathrm{GPa}\right)$ происходит размягчение поперечной моды $T_{1}$ в направлении $\Sigma$. Обсуждается поведение модулей упругости Фукса второго порядка сжатых кристаллов инертных газов.
\end{abstract}

DOI: 10.21883/FTT.2019.01.46906.118

\section{1. Введение}

Наиболее удобными объектами для изучения ряда фундаментальных проблем физики твердого тела являются кристаллы инертных газов (КИГ) - $\mathrm{Ne}, \mathrm{Ar}, \mathrm{Kr}$ и Хе, поскольку они представляют собой относительно простую систему: состоят из атомов с замкнутыми электронными оболочками и содержат один атом в элементарной ячейке. Кроме того, из-за своих структурных и упругих свойств они являются хорошими моделями для изучения фазовых переходов в твердом теле под давлением и сейсмической нестабильности в земной коре и планетах, в особенности газовых планетах-гигантов (Юпитер и др.).

Интенсивное экспериментальное изучение атомных свойств сжатых КИГ началось в конце прошлого века и связано с развитием технологий, позволяющих в лабораторных условиях добиваться высоких давлений $[1,2]$. Применение для изучения фононных спектров метода неупругого рассеяния рентгеновских лучей (inelastic x-ray scattering - IXS) вместо спектроскопических методов неупругого нейтронного рассеяния дает возможность использовать технику ячеек алмазных наковален (diamond-anvil cell - DAC) и поэтому расширить диапазон давлений до $100 \mathrm{GPa}$ и выше (см. обзор [3]). Одним из первых методом IXS в DAC был изучен кристаллический $\mathrm{Ar}$ при давлении до $20 \mathrm{GPa}$ [4].

Как известно, все КИГ (кроме Не) имеют гранецентрированную кубическую (ГЦК) структуру при нормальном давлении стабильную до $100 \mathrm{GPa}$ [5]. В от- личие от других КИГ кристаллический $\mathrm{Ne}$ сохраняет ГЦК-структуру вплоть до давления металлизации, которое оценивается от 1 ТРа до $176 \mathrm{TPa}$ (см. [6,7] и ссылки там). Используя технику ячеек алмазных наковален, $\mathrm{Ar}$ был сжат при комнатной температуре до $80 \mathrm{GPa}$ без изменения в структуре $[8,9]$. В работе $[10]$ на основе теории функционала плотности (density functional theory DFT) авторы предсказывают для Ar переход из ГЦК в гексагональную плотноупакованную (ГПУ) структуру приблизительно при $220 \mathrm{GPa}$, стабильную до $2 \mathrm{TPa}$. Металлизация ГПУ-Ar предсказана вблизи $510 \mathrm{GPa}$ [10]. Исследование $\mathrm{Kr}$ с помощью современной техники DAC до $55 \mathrm{GPa}$ не показало структурных фазовых переходов $[11,12]$. Теоретические расчеты предсказывают для $\mathrm{Kr}$ ГЦК-ГПУ-переход при $130 \mathrm{GPa}$ и металлизацию при $p_{m}=310 \mathrm{GPa}[13,10]$. Эксперимент показал, что Хе под действием давления переходит в ГПУ структуру при $75 \mathrm{GPa}$ [14], а металлизация происходит при $132 \mathrm{GPa}[15]$.

В более поздних экспериментальных исследованиях наблюдается сосуществование ГЦК- и ГПУ-структур от 1.5 до $41 \mathrm{GPa}$ в Хе, от 3.2 до $50 \mathrm{GPa}$ в $\mathrm{Kr}$ и выше 49.6 GPa в $\mathrm{Ar}[16,17,18]$. Проблема ГЦК-ГПУ перехода в нанокластерах $\mathrm{Ar}$ обсуждается также в работе [19]. Рентгеновские измерения, проведенные в 2010 г. показали, что в $\mathrm{Ne}$ сохраняется стабильность ГЦК-фазы до $237 \mathrm{GPa}[20]$.

Теоретические исследования уравнений состояний, энергетических спектров фононов, структурных фазовых переходов, индуцированных давлением, в КИГ и 
других кристаллах ведутся в нескольких направлениях: 1) симметрийный анализ на основе теории групп (см., например, $[21,22]) ; 2$ ) расчеты на основе полуэмпирических моделей (см., например, [23,24,25,26,27]); 3) $a b$ initio расчеты (см. [24,28,29,30,31,32] и ссылки там). Результаты работы из первого направления абсолютно надежны и не ограничены давлением и температурой, но они в принципе не дают численных значений. Расчеты на основе полуэмпирических моделей достаточно просты и наглядны, но их надежность ограничена тем давлением, до которого справедливы, использованные при их получении, экспериментальные величины и соотношения. Отличительной чертой $a b$ initio расчетов (например, на основе DFT) является их предсказательная ценность и потому они всегда актуальны для описания динамических и термодинамических свойств веществ при высоких давлениях.

Это разделение в значительной мере условно. Для получения численных результатов исследования из первого направления комбинируются с современными методами DFT, а расчетные методы опираются на симметрийный анализ. В $a b$ initio расчетах на основе DFT используется столько приближений (особенно для обменно-корреляционного потенциала) и подгоночных параметров, что строго говоря, эти расчеты уже мало отличаются от полуэмпирических [31].

Основная тенденция теоретических исследований сжатых КИГ в последнее десятилетие определяется стремлением добиться точности в расчетах сравнимой с экспериментальной точностью, что невозможно без учета многочастичного взаимодействия [32]. Одним из тестов в этом случае могло бы служить адекватное воспроизведение экспериментальной нерегулярной барической зависимости отклонения от соотношения Коши $\delta(p)$ в ряду кристаллов $\mathrm{Ne}-$ Хе. Однако, как указали авторы [18], ни одна из существующих к тому времени теорий не справилась с этой задачей. Учет трехчастичного взаимодействия и деформации электронных оболочек атомов в квадрупольном приближении в наших расчетах позволил получить зависимость $\delta(p)$ индивидуально для каждого из КИГ в очень хорошем согласии с экспериментом (см. [33] и ссылки там).

В работе [34] на основе неэмпирической версии модели Толпыго, явно учитывающей деформацию электронных оболочек в дипольном приближении, и короткодействующего потенциала с учетом трехчастичного взаимодействия, исследовались фононные дисперсионные кривые сжатых кристаллов ряда $\mathrm{Ne}-\mathrm{Xе}$. Было показано, что при больших сжатиях происходит „размягчение“ критических колебаний, которое приводит к динамической нестабильности ГЦК-решетки этих кристаллов. Ab initio расчет уравнений состояния КИГ, выполненный также на основе неэмпирического короткодействующего потенциала с учетом трехчастичного взаимодействия [35] позволяет достаточно точно определить критическое давление, приводящее к абсолютной неустойчивости ГЦК-решетки кристаллов инертных газов.
Исследование абсолютных неустойчивостей представляет собой интерес в тех случаях, когда фазовые переходы первого рода, которые могут предшествовать абсолютной неустойчивости структуры, близки к ней.

Цель настоящей работы - исследовать влияние трехчастичного взаимодействия и деформации электронных оболочек на структурные переходы и динамическую нестабильность в КИГ при высоких давлениях.

\section{2. Фононные частоты кристаллов инертных газов с учетом трехчастичных сил и деформации электронных оболочек в дипольном приближении}

При структурных ГЦК-ГПУ-переходах в кристаллах $\mathrm{Ar}, \mathrm{Kr}$, Хе ГЦК-решетка становится менее энергетически выгодной, чем ГПУ, но остается еще устойчивой. Здесь мы рассмотрим случай, когда ГЦК-решетка становится абсолютно неустойчивой, то есть когда исчезает минимум ее потенциальной энергии.

Условие устойчивости решетки кристалла относительно механических воздействий состоит в следующем: необходимо, чтобы ее энергия представляла собой положительно определенную квадратичную форму при разложении деформации в ряд по параметрам [36]. Известно, что энергия решетки при однородной деформации является квадратичной формой модулей упругости $C_{\alpha \beta}$ и решетка устойчива относительно однородной деформации, если

$$
\frac{1}{2} C_{\alpha \beta} u_{\alpha} u_{\beta}>0,
$$

и относительно неоднородной деформации, если

$$
\frac{1}{2} D_{\alpha \beta}(\mathbf{q}) u_{\alpha}^{\mathbf{q}} u_{\beta}^{-\mathbf{q}}>0 \text {, }
$$

где $D_{\alpha \beta}-$ динамическая матрица; $u_{i}$ - параметр деформации.

В общем случае произвольной деформации условие (2) эквивалентно требованию положительности квадрата фононных частот. Мнимость частоты означает, что смещения атомов решетки под действием малой деформации будут экспоненциально (а не периодически) изменяться со временем. Для потенциальной энергии кристалла размягчение „критических“ колебаний соответствует уплощению ее минимума, определяющего колебания атомов возле положения равновесия, абсолютная неустойчивость - вырождению минимума в прямую, мнимость частоты - образованию максимума энергии.

На основе $a b$ initio расчетов фононных частот КИГ в модели деформируемых и поляризуемых атомов (модель Толпыго) проведем исследование характера абсолютной неустойчивости и влияния на него деформации электронных оболочек и трехчастичного взаимодействия. 
В модели Толпыго потенциальная энергия $U$ решетки получена в виде (см. обзор [33] и ссылки там)

$$
\begin{aligned}
U= & \min \bar{H}=\mathrm{const}+\sum_{l}\left\{\frac{\left(\mathbf{P}^{l}\right)^{2}}{2 \alpha}+\boldsymbol{\beta}^{l} \cdot \mathbf{P}^{l}-\frac{1}{2} \sum_{l^{\prime}} \frac{C}{\left|\mathbf{r}^{l l^{\prime}}\right|^{6}}\right. \\
& \left.+\frac{1}{2} \sum_{l^{\prime}} K\left(\mathbf{P}^{l}, \mathbf{P}^{l^{\prime}}\right)+\frac{1}{2} \sum_{l^{\prime}}^{n . n \cdot} U_{s r}\left(\left|\mathbf{r}^{l}-\mathbf{r}^{l^{\prime}}\right|\right)\right\}
\end{aligned}
$$

Первые два члена описывают деформацию электронных оболочек в дипольном приближении $(\alpha-$ коэффициент дипольной поляризуемости). Следующий член дает силы Ван-дер-Ваальса. $K$ - кулоновское (в классическом смысле) взаимодействие всех диполей $\mathbf{P}^{l}$ между собой. Последнее слагаемое в (3) представляет короткодействующее отталкивание $E_{s r}$.

Уравнения колебаний решетки для смещений атомов $\mathbf{u}^{l}$ и их дипольных моментов $\mathbf{P}^{l}$ запишутся как

$$
m \ddot{u}_{\alpha}^{l}=-\frac{\partial U}{\partial u_{\alpha}^{l}}, \quad \frac{\partial U}{\partial P_{\alpha}^{l}}=0,
$$

где $m$ - масса электрона.

В работе [37] предложен метод представления короткодействующего потенциала отталкивания $E_{s r}$ в виде разложения по степеням малого параметра - интеграла перекрытия волновых функций электронов соседних атомов $S$.

$$
\begin{aligned}
E_{s r}= & E^{(0)}\left(S^{2}\right)+W_{2}\left(S^{2}\right)+W_{3}\left(S^{3}\right) \\
& +W_{4}\left(S^{4}\right)+W_{5}\left(S^{5}\right)+W_{6}\left(S^{6}\right) .
\end{aligned}
$$

Здесь первое $E^{(0)}$ и второе $W_{2}$ слагаемые содержат только одно- и двухцентровые интегралы и соответствуют парному взаимодействию. Слагаемое $W_{3}-$ поправка третей степени по $S$, которое содержит также трехцентровые интегралы и соответствует трехчастичным взаимодействиям. Поправка четвертой степени по $S-W_{4}-$ смешанного типа. Она состоит из одно-, двух-, трех- и четырехцентровых интегралов. Поправка пятой степени $W_{5}$ состоит только из трехцентровых интегралов, а поправка шестой степени $W_{6}$ - только из двухцентровых. Каждый из перечисленных интегралов умножается на $S$ в соответствующей степени.

Двухцентровые кулоновские интегралы были точно рассчитаны на основе таблиц Клементи и Роетти [38]. Полученные при этом закономерности были использованы для аппроксимации трех- и четырехцентровых интегралов произведениями соответствующих интегралов перекрытия.

Тогда для случая, когда атомы $l, l^{\prime}, l^{\prime \prime}$ образуют равносторонний треугольник и при $S \ll 1$, трехчастичный потенциал $W_{3}$ можно привести к простой форме, а именно

$$
\begin{gathered}
W_{3}=-\sum_{l l^{\prime} l^{\prime \prime}}\left(S\left(r^{l l^{\prime \prime}}\right)\right)^{2} f\left(r_{1}\right), \\
f\left(r_{1}\right)=\frac{S\left(r_{1}\right)}{r_{1}}, \quad r_{1}=\left|\mathbf{r}^{l^{\prime}}-\frac{1}{2} \mathbf{r}^{l l^{\prime \prime}}\right|,
\end{gathered}
$$

где $S=S_{n p_{z} n p_{z}}^{l l^{\prime}}$ - наибольший из интегралов перекрытия между внешними $n p$-орбиталями электронов. В отличие от парного потенциала $W_{2}\left(r^{l l^{\prime}}\right)$, трехчастичный потенциал $W_{3}$ зависит не только от $r^{l l^{\prime}}$ и $r^{l l^{\prime \prime}}$, но и от $\left(\mathbf{r}^{l l^{\prime}} \cdot \mathbf{r}^{l l^{\prime \prime}}\right)$. Аргумент функции $f\left(r_{1}\right)$ представляет собой медиану рассматриваемого треугольника $l, l^{\prime}, l^{\prime \prime}$. В данном случае $r_{1}=r^{l l^{\prime}} \cdot \cos \frac{\pi}{6}=a \frac{\sqrt{6}}{2}$. В случае всестороннего сжатия углы не меняются, изменяется только постоянная решетки $a$.

Выполняя дифференцирование в уравнениях движения (4), подставляя все переменные $\mathbf{p}^{l}=e \mathbf{u}^{l}, \mathbf{P}^{l}$ в виде плоских волн $\exp \{i \mathbf{k r}-i \omega t\}$ и суммируя по $l^{\prime} l^{\prime \prime}$, можно получить уравнения для амплитуд $p_{\alpha}, P_{\alpha}$ с учетом рассмотренного трехчастичного взаимодействия.

В уравнениях колебаний решетки наряду с короткодействующими трехчастичными силами, обусловленными перекрытием электронных оболочек атомов [37], будем рассматривать также трехчастичное взаимодействие, обязанное деформации электронных оболочек. В работе [34] с учетом всех рассмотренных трехчастичных сил были приведены выражения для квадратов собственных частот в симметричных направлениях волнового вектора $\mathbf{k}$ (безразмерные переменные $\left.\Omega=\omega \sqrt{m a^{3} / e^{2}}, \mathbf{k}=a \mathbf{K}\right)$. Выделим так называемые „критические“ точки $X$ и $L$, и направление $\Sigma$, где происходит „размягчение“ фононных частот. $\hbar \omega_{\lambda \mathbf{k}} \rightarrow 0$.

Для направления $\Delta$, т. X [001]

$$
\begin{aligned}
& \Omega_{L}^{2}=4(G+H)+B \chi_{z z}+4\left[A_{1}-\frac{(2 h+2 g)^{2}}{A^{-1}-\varphi_{z z}}\right] . \\
& \Omega_{T}^{2}=\left(G+2 H+V_{t}\right)+B \chi_{x x}+4\left[B_{1}-\frac{(2 h+g)^{2}}{A^{-1}-\varphi_{x x}}\right] .
\end{aligned}
$$

Для направления $\Lambda$, т. L $[1 / 2,1 / 2,1 / 2]$

$$
\begin{aligned}
\Omega_{L}^{2}=(4 G & \left.+3 H+2 E+6 F+V_{t}\right)+B\left(\chi_{x x}+2 \chi_{x y}\right) \\
& +\left[A_{2}-\frac{(3 h+4 g)^{2}}{A^{-1}-2 \varphi_{x y}}\right] . \\
\Omega_{T}^{2}= & (G+3 H+2 E+6 F)+B\left(\chi_{x x}-2 \chi_{x y}\right) \\
& +\left[B_{2}-\frac{(3 h+g)^{2}}{A^{-1}+\varphi_{x y}}\right] .
\end{aligned}
$$

Для направления $\Sigma(\mathbf{k} \|[110])$

$$
\begin{aligned}
\Omega_{T_{1}}^{2} & =(H+2 E+4 F) \sin ^{2} k_{x}+(G+2 H)\left(1-\cos k_{x}\right) \\
& +\left[D_{1}-\frac{h^{2}}{A^{-1}-\varphi_{x x}+\varphi_{x y}}\right] \sin ^{4} k_{x} \\
& +\left[D_{2}-\frac{2 h(2 h+g)}{A^{-1}-\varphi_{x x}+\varphi_{x y}}\right]\left(1-\cos k_{x}\right) \sin ^{2} k_{x} \\
& +\left[D_{3}-\frac{(2 h+g)^{2}}{A^{-1}-\varphi_{x x}+\varphi_{x y}}\right]\left(1-\cos k_{x}\right)^{2}+B\left(\chi_{x x}-\chi_{x y}\right) .
\end{aligned}
$$

Здесь $H=H_{0}+\delta H, G=G_{0}+\delta G, H_{0}(a \sqrt{2})$ и $G_{0}(a \sqrt{2})$ являются первой и второй производными короткодействующего парного потенциала отталкивания для равновесных расстояний первых соседей; аналогично для 

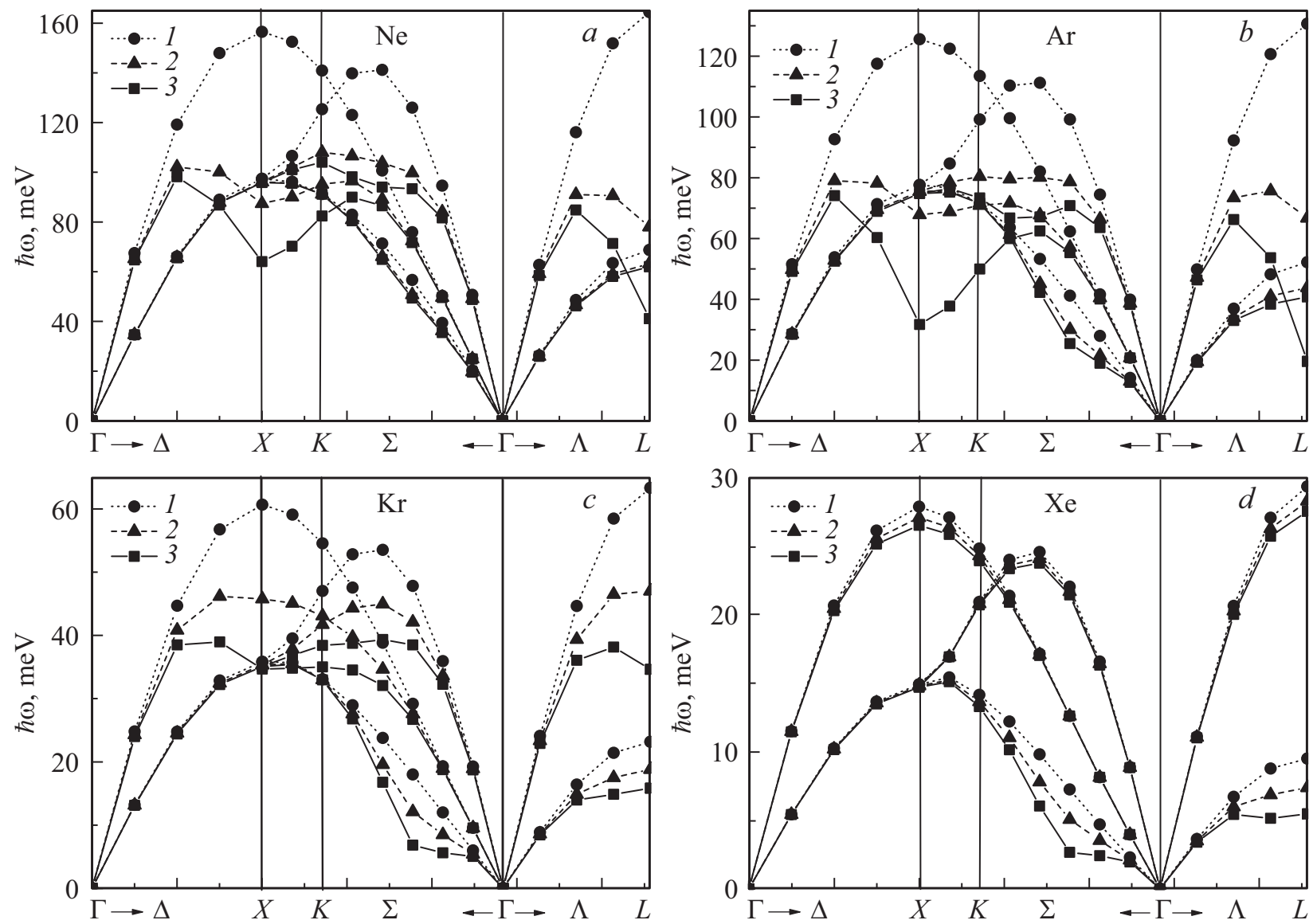

Рис. 1. Фононные дисперсионные кривые в симметричных направлениях волнового вектора $\mathbf{k}$ для Ne (a) при сжатии $u=\Delta V / V_{0}=0.76$, для $\operatorname{Ar}(b)$ при $u=0.71$, для $\operatorname{Kr}(c)$ при $u=0.68$ и для Хе $(d)$ при $u=0.6 .1$ - расчеты в модели МТ 0 с учетом трехчастичных сил за счет перекрытия электронных оболочек, но без учета деформации электронных оболочек; 2 - расчеты в модели $\mathrm{MT}_{1}$ с учетом трехчастичных сил за счет перекрытия электронных оболочек и деформации электронных оболочек в парном приближении; 3 - расчеты в модели $\mathrm{MT}_{2}$ с учетом трехчастичных сил и деформации электронных оболочек в парном и трехчастичном приближениях.

вторых соседей $F=H_{0}(2 a)$ и $E=G_{0}(2 a) ; B$ определяет взаимодействие Ван-дер-Ваальса; $h$ и $g$ - параметры деформации электронных оболочек атомов дипольного типа в парном приближении; $\chi_{x x}, \chi_{x y}, \chi_{x z}-$ функции $\mathbf{k}$, происходящие от ван-дер-ваальсовских сил; $\varphi_{x x}, \varphi_{x y}, \varphi_{x z}$ - коэффициенты электрического поля, вызванного системой диполей $\mathbf{P}^{l} ; A-$ безразмерная поляризуемость атома (см. [33] и ссылки там). Параметры $\delta G, \delta H$ и $V_{t}$ описывают трехчастичные короткодействующие силы, обусловленные перекрытием электронных оболочек (недеформированных) атомов [37]. Как мы видим, влияние этого трехчастичного взаимодействия сказывается при всех $\mathbf{k}$, включая величину наклона ветвей при $\mathbf{k} \rightarrow 0$. Параметры $A_{i}, B_{i}, D_{i}$ описывают трехчастичные силы, связанные со взаимной деформацией электронных оболочек. Эти слагаемые фактически не дают новой зависимости от $\mathbf{k}$ по сравнению с рассмотренной ранее „парной“ деформацией электронных оболочек в дипольном приближении (и не дают вклада в упругие модули). Параметры, необходимые для расчета фононных частот в зависимости от сжатия $u=\Delta V / V_{0}$
$\left(\Delta V=V_{0}-V(p \neq 0), V_{0}\right.$ - объем при $\left.p=0\right)$ представлены в работе [34].

\section{3. Расчет фононных частот при сильных сжатиях и обсуждение}

На pис. $1, a-d$ представлены фононные частоты $\hbar \omega_{\lambda \mathbf{k}}$ в симметричном направлении волнового вектоpa k для $\mathrm{Ne}(u=0.76), \operatorname{Ar}(u=0.71), \operatorname{Kr}(u=0.68)$ и Хе $(u=0.6)$. Три группы кривых соответствуют трем вариантам (моделям) расчетов $\hbar \omega_{\lambda \mathbf{k}}$ : в модели $M T_{0}$ учитывается трехчастичное взаимодействие за счет перекрытия электронных оболочек атомов $(\delta G \neq 0$, $\left.\delta H \neq 0, V_{t} \neq 0\right)$, но не рассматривается их деформация $\left(A_{i}=B_{i}=D_{i}=0, g=h=0\right)$; в модели $M T_{1}$ добавляется учет деформации электронных оболочек в ,парном“ приближении $\left(A_{i}=\ldots=D_{i}=0, g \neq 0, h \neq 0\right)$; в модели $M T_{2}$ учитываются все рассмотренные трехчастичные силы и деформация электронных оболочек атомов $\left(\delta G \neq 0, \delta H \neq 0, V_{i} \neq 0, A_{i} \neq 0 \ldots D_{i} \neq 0, g \neq 0, h \neq 0\right)$. 
Частоты $\hbar \omega_{\lambda \mathbf{k}}[\mathrm{meV}]$ в моделях $M T_{0}(M 2)$ и $M T_{2}(M 2 a)$ и относительный вклад трехчастичного взаимодействия и эффектов деформации электронных оболочек $\gamma[\%]$ при различных степенях сжатия для КИГ в „критических“ точках

\begin{tabular}{|c|c|c|c|c|c|c|c|c|c|c|}
\hline \multicolumn{2}{|l|}{ Теория } & \multicolumn{3}{|c|}{$\hbar \omega_{\lambda \mathbf{k}}$ в модели $M T_{0}(M 2)$ в модели $M T 2$} & \multicolumn{3}{|c|}{$\hbar \omega_{\lambda \mathbf{k}}$ в модели $M T_{2}(M 2 a)$} & \multicolumn{3}{|c|}{$\gamma, \%$} \\
\hline \multicolumn{11}{|c|}{$\mathrm{Ne}$} \\
\hline \multicolumn{2}{|l|}{$u$} & 0.3 & 0.7 & 0.76 & 0.3 & 0.7 & 0.76 & 0.3 & 0.7 & 0.76 \\
\hline \multirow{2}{*}{\multicolumn{2}{|c|}{$\mathbf{k}, \lambda$}} & \multicolumn{9}{|c|}{$p, \mathrm{GPa}$} \\
\hline & & 1.886 & 169.62 & 441.47 & 1.886 & 169.62 & 441.47 & 1.886 & 169.62 & 441.47 \\
\hline$X[0 ; 0 ; 1]$ & $\begin{array}{l}L \\
T\end{array}$ & $\begin{array}{c}18.18 \\
(18.12) \\
11.9 \\
(11.94)\end{array}$ & $\begin{array}{c}105.99 \\
(105.71) \\
66.561 \\
(67.05)\end{array}$ & $\begin{array}{l}156.51 \\
97.389\end{array}$ & $\begin{array}{c}17.966 \\
(17.91) \\
11.87 \\
(11.92)\end{array}$ & $\begin{array}{l}79.594 \\
(64.68) \\
65.626 \\
(69.52)\end{array}$ & $\begin{array}{l}64.059 \\
95.871\end{array}$ & $\begin{array}{c}1.18 \\
(1.14) \\
0.25 \\
(0.23)\end{array}$ & $\begin{array}{c}24.9 \\
(38.8) \\
1.4 \\
(3.67)\end{array}$ & $\begin{array}{l}59.07 \\
1.56\end{array}$ \\
\hline$L[1 / 2 ; 1 / 2 ; 1 / 2]$ & $\begin{array}{l}\mathrm{L} \\
T\end{array}$ & $\begin{array}{c}18.81 \\
(18.33) \\
8.59 \\
(7.65)\end{array}$ & $\begin{array}{c}110.9 \\
(108.13) \\
46.79 \\
(41.03)\end{array}$ & $\begin{array}{l}164.45 \\
68.731\end{array}$ & $\begin{array}{c}18.559 \\
(18.11) \\
8.586 \\
(7.65)\end{array}$ & $\begin{array}{l}79.105 \\
(74.7) \\
45.616 \\
(42.81)\end{array}$ & $\begin{array}{l}41.147 \\
62.017\end{array}$ & $\begin{array}{c}1.33 \\
(1.21) \\
0.05 \\
(0.01)\end{array}$ & $\begin{array}{c}28.67 \\
(30.92) \\
2.51 \\
(4.34)\end{array}$ & $\begin{array}{l}74.98 \\
9.77\end{array}$ \\
\hline \multicolumn{11}{|c|}{$\mathrm{Ar}$} \\
\hline \multicolumn{2}{|l|}{$u$} & 0.3 & 0.6 & 0.71 & 0.3 & 0.6 & 0.71 & 0.3 & 0.6 & 0.71 \\
\hline \multirow{2}{*}{\multicolumn{2}{|c|}{$\mathbf{k}, \lambda$}} & \multicolumn{9}{|c|}{$p, \mathrm{GPa}$} \\
\hline & & 4.398 & 97.918 & 405.195 & 4.398 & 97.918 & 405.195 & 4.398 & 97.918 & 405.195 \\
\hline$X[0 ; 0 ; 1]$ & $\begin{array}{l}L \\
T\end{array}$ & $\begin{array}{l}21.492 \\
(21.27) \\
13.761 \\
(13.99)\end{array}$ & $\begin{array}{l}69.107 \\
(68.59) \\
42.955 \\
(43.79)\end{array}$ & $\begin{array}{l}125.55 \\
77.622\end{array}$ & $\begin{array}{c}20.893 \\
(20.73) \\
13.695 \\
(13.84)\end{array}$ & $\begin{array}{l}55.695 \\
(53.86) \\
42.145 \\
(45.08)\end{array}$ & $\begin{array}{l}31.735 \\
74.805\end{array}$ & $\begin{array}{c}2.79 \\
(2.53) \\
0.48 \\
(1.06)\end{array}$ & $\begin{array}{c}19.41 \\
(21.47) \\
1.89 \\
(2.93)\end{array}$ & $\begin{array}{l}74.7 \\
3.63\end{array}$ \\
\hline$L[1 / 2 ; 1 / 2 ; 1 / 2]$ & $\begin{array}{l}L \\
T\end{array}$ & $\begin{array}{c}21.887 \\
(21.54) \\
9.105 \\
(8.97) \\
\end{array}$ & $\begin{array}{l}71.398 \\
(70.05) \\
28.462 \\
(27.06)\end{array}$ & $\begin{array}{c}130.668 \\
52.217\end{array}$ & $\begin{array}{l}21.218 \\
(21.06) \\
9.103 \\
(8.96) \\
\end{array}$ & $\begin{array}{l}56.461 \\
(57.81) \\
27.688 \\
(27.61) \\
\end{array}$ & $\begin{array}{l}19.589 \\
40.825\end{array}$ & $\begin{array}{c}3.06 \\
(2.41) \\
0.02 \\
(0.12)\end{array}$ & $\begin{array}{c}20.92 \\
(17.47) \\
2.72 \\
(2.03)\end{array}$ & $\begin{array}{c}85 \\
21.8\end{array}$ \\
\hline $\mathbf{k}[3 / 8 ; 3 / 8 ; 0]$ & $T_{1}$ & 6.941 & 22.031 & 41.194 & 6.949 & 20.918 & 25.445 & 0.11 & 5.05 & 38.2 \\
\hline \multicolumn{11}{|c|}{$\mathrm{Kr}$} \\
\hline \multicolumn{2}{|l|}{$u$} & 0.3 & 0.6 & 0.68 & 0.3 & 0.6 & 0.68 & 0.3 & 0.6 & 0.68 \\
\hline \multirow{2}{*}{\multicolumn{2}{|c|}{$\mathbf{k}, \lambda$}} & \multicolumn{9}{|c|}{$p, \mathrm{GPa}$} \\
\hline & & 5.771 & 93.455 & 222.995 & 5.771 & 93.455 & 222.995 & 5.771 & 93.455 & 222.995 \\
\hline$X[0 ; 0 ; 1]$ & $\begin{array}{l}L \\
T\end{array}$ & $\begin{array}{l}15.220 \\
(14.94) \\
9.461 \\
(9.77) \\
\end{array}$ & $\begin{array}{l}42.390 \\
(41.85) \\
25.162 \\
(26.23) \\
\end{array}$ & $\begin{array}{l}60.684 \\
35.811\end{array}$ & $\begin{array}{c}14.577 \\
(14.6) \\
9.373 \\
(9.62) \\
\end{array}$ & $\begin{array}{l}33.198 \\
(36.45) \\
24.66 \\
(26.6) \\
\end{array}$ & $\begin{array}{l}34.671 \\
35.063\end{array}$ & $\begin{array}{c}4.22 \\
(2.31) \\
0.93 \\
(1.45) \\
\end{array}$ & $\begin{array}{c}21.68 \\
(12.89) \\
2 \\
(1.4) \\
\end{array}$ & $\begin{array}{l}42.87 \\
2.09\end{array}$ \\
\hline$L[1 / 2 ; 1 / 2 ; 1 / 2]$ & $\begin{array}{l}L \\
T\end{array}$ & $\begin{array}{c}15.504 \\
(15.15) \\
6.164 \\
(6.19)\end{array}$ & $\begin{array}{l}43.965 \\
(42.86) \\
16.192 \\
(15.65)\end{array}$ & $\begin{array}{l}63.337 \\
23.172\end{array}$ & $\begin{array}{c}14.807 \\
(14.83) \\
6.163 \\
(6.18)\end{array}$ & $\begin{array}{l}33.889 \\
(38.28) \\
15.142 \\
(15.96)\end{array}$ & $\begin{array}{l}34.627 \\
15.830\end{array}$ & $\begin{array}{c}4.49 \\
(2.12) \\
0.02 \\
(0.18)\end{array}$ & $\begin{array}{c}22.92 \\
(10.69) \\
6.48 \\
(1.98)\end{array}$ & $\begin{array}{l}45.33 \\
31.68\end{array}$ \\
\hline $\mathbf{k}[3 / 8 ; 3 / 8 ; 0]$ & $T_{1}$ & 4.632 & 12.349 & 17.989 & 4.625 & 10.887 & 6.809 & 0.51 & 11.84 & 62.15 \\
\hline \multicolumn{11}{|c|}{$\mathrm{Xe}$} \\
\hline \multicolumn{2}{|l|}{$u$} & 0.3 & 0.5 & 0.6 & 0.3 & 0.5 & 0.6 & 0.3 & 0.5 & 0.6 \\
\hline \multirow{2}{*}{\multicolumn{2}{|c|}{$\mathbf{k}, \lambda$}} & \multicolumn{9}{|c|}{$p, \mathrm{GPa}$} \\
\hline & & 7.215 & 36.603 & 84.429 & 7.215 & 36.603 & 84.429 & 7.215 & 36.603 & 84.429 \\
\hline$X[0 ; 0 ; 1]$ & $\begin{array}{l}L \\
T\end{array}$ & $\begin{array}{c}12.462 \\
(12.12) \\
7.456 \\
(7.87)\end{array}$ & 11.807 & 14.933 & $\begin{array}{c}12.319 \\
(11.92) \\
7.441 \\
(7.82)\end{array}$ & $\begin{array}{l}20.339 \\
11.810\end{array}$ & $\begin{array}{l}26.546 \\
14.731\end{array}$ & $\begin{array}{c}1.15 \\
(1.64) \\
0.2 \\
(0.61)\end{array}$ & $\begin{array}{l}3.63 \\
0.03\end{array}$ & $\begin{array}{l}4.87 \\
1.35\end{array}$ \\
\hline$L[1 / 2 ; 1 / 2 ; 1 / 2]$ & $\begin{array}{l}L \\
T\end{array}$ & $\begin{array}{c}12.691 \\
4.752\end{array}$ & $\begin{array}{c}21.885 \\
7.402\end{array}$ & $\begin{array}{c}29.374 \\
9.515\end{array}$ & $\begin{array}{c}12.415 \\
4.736\end{array}$ & $\begin{array}{c}20.939 \\
6.872\end{array}$ & $\begin{array}{c}27.572 \\
5.478\end{array}$ & $\begin{array}{l}2.17 \\
0.34\end{array}$ & $\begin{array}{l}4.32 \\
7.16\end{array}$ & $\begin{array}{c}6.13 \\
42.43\end{array}$ \\
\hline $\mathbf{k}[3 / 8 ; 3 / 8 ; 0]$ & $T_{1}$ & 3.476 & 5.433 & 7.254 & 3.452 & 4.863 & 2.673 & 0.69 & 10.49 & 63.15 \\
\hline
\end{tabular}

Примечание. $\gamma=\left[\left(\hbar \omega\left(M T_{0}\right)-\hbar \omega\left(M T_{2}\right)\right) / \hbar \omega\left(M T_{0}\right)\right] \cdot 100 \%$. (В скобках даны значения частот рассчитанных в моделях М2 и М2а и соответствующие значения $\gamma[39])$. 

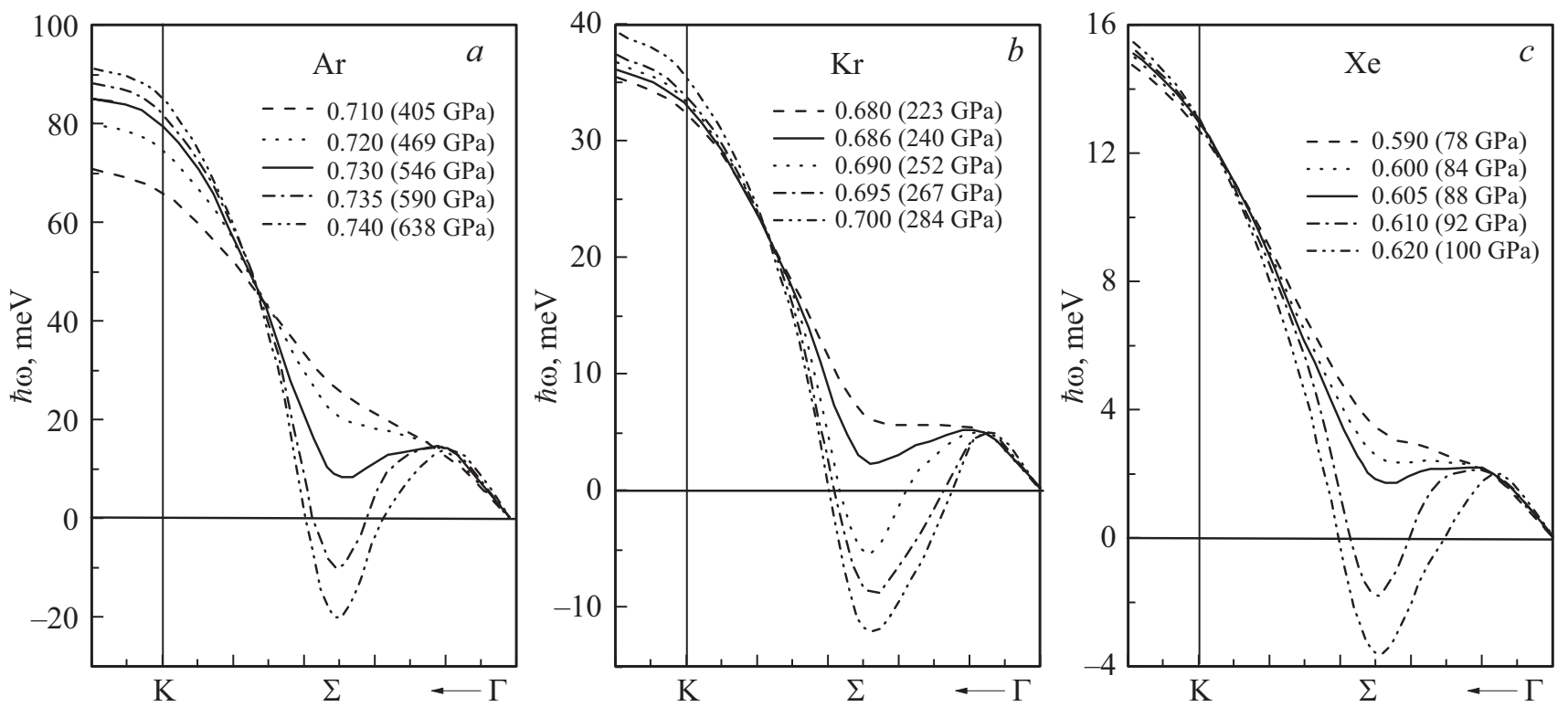

Pис. 2. Фононная дисперсия частот поперечной ветви $\mathrm{T}_{1}$ в направлении $\Sigma$ для $\mathrm{Ar}, \mathrm{Kr}$ и $\mathrm{Xe}$ при различных сжатиях $u=\Delta V / V_{0}$ (давлениях $p$ ).

Как ожидалось, наибольшее „размягчение“фононных частот получается при учете деформации электронных оболочек в парном и трехчастичном приближениях, причем для $\mathrm{Ne}, \mathrm{Ar}, \mathrm{Kr}$ и Хе „размягчаются“ продольные моды в точках $X$ и $L$. В $\mathrm{Kr}$ и Хе наряду с указанным „размягчением“ наблюдается размягчение поперечной моды $T_{1}$ в направлении [110] и вырожденной поперечной моды $T$ в точке $L$.

В таблице представлены численные значения $\hbar \omega_{\lambda \mathbf{k}}$ при различном сжатии в тех точках зоны Бриллюэна, где визуально наблюдается (на рис. $1, a-d$ ) наибольшее „размягчение“ фононных частот. Как видно из таблицы наибольший вклад деформации электронных оболочек наблюдается в продольную моду $\hbar \omega_{\lambda \mathbf{k}}$ в т. $L$ для $\mathrm{Ne}(74.98 \%)$ и $\mathrm{Ar}(66.06 \%)$. Для $\mathrm{Kr}$ этот вклад (45.33\%) сравним с вкладом в симметричном направлении $\Sigma$ (т. $\mathbf{k}[3 / 8 ; 3 / 8 ; 0])$ для поперечной моды $T_{1}(62.17 \%)$. Именно в этой точке для Хе наблюдается наиболее значительный вклад деформации электронных оболочек (63.15\%) и гораздо меньше для поперечной моды $\hbar \omega_{\lambda \mathbf{k}}$ в т. $L$.

Для $\mathrm{Ne}, \mathrm{Ar}, \mathrm{Kr}$ в т. $L$ даны в скобках результаты расчетов энергии фононов $\hbar \omega_{\lambda \mathbf{k}}$ в модели $M 2$ (без учета трехчастичных сил и деформации электронных оболочек) и в модели $M 2 a$ (без трехчастичных сил, но с учетом деформации электронных оболочек в парном приближении) [39]. Сравнение значений $\hbar \omega_{\lambda \mathbf{k}}$ для $\mathrm{Ne}(u=0.7), \operatorname{Ar}(u=0.6)$ и $\operatorname{Kr}(u=0.6)$, полученных в моделях $M T_{0}$ и $M 2$, показывает, что энергия фононов растет при включении трехчастичных сил, обязанных перекрытию электронных оболочек для всех сжатий. Результаты расчетов в моделях $M T_{2}$ и $M 2 a$ показывают уменьшение $\hbar \omega_{\lambda \mathbf{k}}$ при учете трехчастичных сил за счет деформации электронных оболочек, которое компенсируется ростом в модели $M T_{0}$. Таким образом, получаются значения $\gamma$ близкие к рассчитанным ранее с парным неэмпирическим потенциалом [39].

На рис. 2, $a-c$ проиллюстрировано размягчение „критических“ колебаний поперечной ветви $T_{1}$ в направлении $\Sigma$ для $\mathrm{Ar}, \mathrm{Kr}$ и Хе. Сплошной линией мы обозначили рассчитанные минимальные значения $\hbar \omega_{\lambda \mathbf{k}}(u)$ при указанных на рис. 2 сжатиях. Будем считать эти сжатия и соответствующие им давления критическими $p_{\mathrm{c}}$. При больших сжатиях частота становиться мнимой. Как видно из рис. $2, b, c$ при давлениях больше критических $p>p_{\text {c }}$ и соответствующих им сжатиях равным 0.686 $\left(p_{c}=240 \mathrm{GPa}\right)$ для $\mathrm{Kr}$ и $0.605\left(p_{c}=88 \mathrm{GPa}\right)$ для $\mathrm{Xe}$, $\hbar \omega_{\lambda \mathbf{k}} \rightarrow 0$ и наступает абсолютная неустойчивость. В $\mathrm{Ar}$ частоты обращаются в нуль быстрее на границе зоны Бриллюэна в т. $L[1 / 2 ; 1 / 2 ; 1 / 2]$ в отличие от $\mathrm{Kr}$ и Хе. Как показывают расчеты, представленные в таблице, в $\mathrm{Ar}$ размягчается продольная мода $L$ в т. $L$ при сжатии 0.71 $\left(p_{\mathrm{c}}=405 \mathrm{GPa}\right)$ и в $\mathrm{Ne}$ при сжатии $0.76\left(p_{\mathrm{c}}=442 \mathrm{GPa}\right)$.

\section{4. Поведение модулей упругости второго порядка сжатых кристаллов инертных газов}

Если пренебречь тем, что произошел фазовый ГЦК-ГПУ-переход, то как показано в предыдущем пунк-

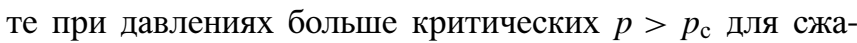
тий $u$ равных $0.605\left(p_{\mathrm{c}}=88 \mathrm{GPa}\right)$ и $0.686\left(p_{\mathrm{c}}=240 \mathrm{GPa}\right)$ для Хе и $\mathrm{Kr}$ соответственно наблюдается обращение в нуль частоты „критической“ ветви $T_{1}$ в точке $\mathbf{k}[3 / 8 ; 3 / 8 ; 0]$ (рис. $2, b, c)$, т.е. наступает абсолютная неустойчивость, указывающая на необходимость фазового перехода. Для легких кристаллов $\mathrm{Ne}$ и $\mathrm{Ar}$ стремление $\hbar \omega_{\lambda \mathbf{k}}$ к нулю для сжатий $0.76\left(p_{\mathrm{c}}=442 \mathrm{GPa}\right)$ и $0.71\left(p_{\mathrm{c}}=405 \mathrm{GPa}\right)$ 
соответственно проявилось на границе зоны Бриллюэна в т. $L$ (см. рис. $1, a, b$ и таблицу).

Подчеркнем, что рассмотрение одной лишь механической устойчивости не решает вопрос о реальной структуре кристалла, которая для фазовых переходов, происходящих под действием давления, определяется термодинамическим потенциалом. Как правило, абсолютным неустойчивостям предшествуют фазовые переходы первого рода, поэтому критические сжатия указывают лишь верхнюю границу по сжатию для фазового перехода.

Исходя из общей теории устойчивости кристаллов, можно предположить, что при приближении к структурному фазовому переходу в точке перехода и после нее модули упругости могут претерпевать заметные изменения. Для фазовых переходов второго рода в рамках феноменологической теории можно показать, как будут изменяться модули упругости под действием различных деформаций [40]. Для рассматриваемых структурных фазовых переходов первого рода такой теории не существует, и информацию о поведении модулей упругости при структурном переходе и вблизи него можно получить с помощью неэмпирических расчетов. В связи с этим развитая квантово-механическая модель деформируемых и поляризуемых атомов была применена к исследованию поведения модулей упругости кристаллов инертных газов под действием давления [41].

В работе [41] были рассмотрены модули упругости типа Фукса $B_{i k}$ для ГЦК-кристаллов ряда $\mathrm{Ne}-\mathrm{Xe}$ при $T=0$. Модули упругости типа Фукса $B_{i k}$ удобнее, чем общепринятые модули Браггера $C_{i k}$, поскольку параметры деформации, по которым разлагается свободная энергия, имеют наглядный физический смысл, а именно, можно выделить чисто сдвиговые модули $B_{33}, B_{44}$ и модуль всестороннего сжатия $B_{11}$. Модули Фукса можно представить в виде

$$
B_{i j}=B_{i j}^{(0)}+B_{i j}^{t}+B_{i j}^{q}
$$

$B_{i j}^{(0)}$ - модули упругости Фукса, рассчитанные с парным потенциалом; $B_{i j}^{t}$ - вклад трехчастичного взаимодействия; $B_{i j}^{q}$ описывает эффекты деформации электронных оболочек в квадрупольном приближении. Абсолютные величины $\left|B_{i j}^{t}\right|$ и $B_{i j}^{q}$ растут в ряду $\mathrm{Ne}-$ Хе. Как показал расчет, деформация электронных оболочек не влияет на модуль всестороннего сжатия $B_{11}$, а трехчастичная поправка $B_{11}^{t}$ всегда положительна. Объемная зависимость модуля $B_{11}(u)$, определяющего наклон при $\mathbf{k} \rightarrow 0$ продольных фононных ветвей, достаточно сильная и не нарушает монотонность в ряду $\mathrm{Ne}, \mathrm{Ar}, \mathrm{Kr}, \mathrm{Xe}$. Для сдвиговых модулей $B_{33}$ и $B_{44}$ трехчастичные поправки $B_{33}^{t}<0$ и $B_{44}^{t}<0$ также, как и соответствующие квадрупольные поправки. Поведение сдвигового модуля $B_{33}(u)$ качественно подобно $B_{11}(u)$, но монотонность в ряду $\mathrm{Ne}-\mathrm{Xе} \mathrm{нарушается.} \mathrm{Характер} \mathrm{объемной} \mathrm{зависимости}$ модуля $B_{44}(u)$, определяющего наклоны поперечных фононных ветвей [100] и [110] значительно отличается от $B_{33}(u)$ и $B_{11}(u)$, особенно для Хе (см. рис. $1, c$ в [41]). Это отличие связано с тем, что абсолютная величина вклада трехчастичного взаимодействия $\left|B_{44}^{t}\right|$ в модуль $B_{44}$ в 2 раза больше, чем в $B_{33}$, а вклада квадрупольной деформации $\left|B_{44}^{q}\right|$ больше в 8 раз.

Как показали наши расчеты, в Хе при сжатиях $u=0.6$ сдвиговый модуль $B_{44}$ обращается в нуль, что согласно (1) указывает на появление абсолютной неустойчивости и необходимость фазового перехода. Действительно такой переход был экспериментально обнаружен в [42]. Это переход из ГЦК- в ГПУ-фазу при $75 \mathrm{GPa}$ непосредственно перед металлизацией, происходящей при $u=0.65$ (132 GPa) [15].

\section{5. Заключение}

Анализ поведения сдвиговых модулей Фукса второго порядка $B_{33}(p)$ и $B_{44}(p)$ показал существенную роль эффектов деформации электронных оболочек, которая усиливается с ростом давления. Деформация электронных оболочек не вносит вклада в модуль всестороннего сжатия $B_{11}$, а трехчастичная поправка $B_{11}^{t}$ мала на фоне $B_{11}^{(0)}$, поэтому поведение $B_{11}(p)$ можно описать с помощью эффективного парного потенциала. Этим, на наш взгляд, объясняется хорошее согласие с экспериментом барической зависимости $B_{11}(p)$, рассчитанных в разных эмпирических моделях, и гораздо худший результат для сдвиговых модулей $B_{33}(p)$ и $B_{44}(p)$ (см., например, $[43,33])$. Только при учете трехчастчного взаимодействия и квадрупольной деформации электронных оболочек оказалось возможно адекватно, в хорошем согласии с экспериментом воспроизвести барическую зависимость коэффициента упругой анизотропии Зенера $A_{Z}(p)=B_{44}(p) / B_{33}(p)$ и отклонения от соотношения Коши $\delta(p)$ [33].

Исследование фононных спектров, представленное в настоящей работе, показало, что трехчастичные силы за счет перекрытия электронных оболочек малы даже при больших давлениях и наиболее заметны в Хе. Кроме этого в настоящей работе оценивалась роль трехчастичных сил, обусловленных взаимной деформацией электронных оболочек. Эти эффекты оказались более значительными. Они ярко проявились в ,размягчении“ критических фононных мод во всех кристаллах ряда $\mathrm{Ne}-\mathrm{Xе} \mathrm{при} \mathrm{соответствующих} \mathrm{сжатиях.}$

Как указано во введение, авторы работы [4] ставили своей целью показать принципиальную возможность измерения энергетического спектра фононов при больших давлениях методом IXS, используя DAC. Наилучшие результаты с минимальной экспериментальной погрешностью получены для поперечной $\hbar \omega_{T}(\mathbf{k})$ и продольной $\hbar \omega_{L}(\mathbf{k})$ мод в $\mathrm{Ar}$ при давлении $p=3.1 \mathrm{GPa}$ в направлении $\Delta(\mathbf{k} \|[001])$. Второй задачей авторов было сравнить полученные экспериментальные результаты с расчетами используя „чистый“ парный потенциал, эффективный парный потенциал и учитывая трехчастичное взаимодействие по формуле Слетера-Кирквуда. Как 
оказалось результаты расчетов с „чистым“ парным потенциалом отличаются от экспериментальных значений для $\hbar \omega_{T}(X)$ в среднем на $10 \%$. Учет трехчастичного взаимодействия мало меняет поперечную моду, но в продольную моду вносит значительный вклад даже при давлении $p=3.1 \mathrm{GPa}$, что однако недостаточно улучшает согласие для $\hbar \omega_{L}(X)$ (примерно на $1 \%$, а нужно $7 \%)$. По словам авторов, у них не было задачи получить многочастичный потенциал, способный устранить расхождения теории и эксперимента, но по сравнению с уравнением состояния, адекватное описание $\hbar \omega_{\lambda \mathbf{k}}$ может служить весомым аргументом при тестировании многочастичного взаимодействия в системах под действием высокого давления.

Расчет $\hbar \omega_{\lambda}(X)$, выполненный в работе [44] с „чистым“ парным потенциалом (модель $M 2$, без учета деформации электронных оболочек) дает расхождение с экспериментом 2.6\% $\hbar \omega_{L}(X)$ и $8 \%$ для $\hbar \omega_{T}(X)$. Расхождение с экспериментом наших расчетов $\hbar \omega_{\lambda}(X)$ в модели $M T_{0}$ (без учета деформации электронных оболочек, но с учетом трехчастичного взаимодействия, обусловленного перекрытием электронных оболочек) составляет $3.7 \%$ для $\hbar \omega_{L}(X)$ и $6.4 \%$ для $\hbar \omega_{T}(X)$. Учет трехчастичного взаимодействия и деформации электронных оболочек в парном и трехчастичном приближениях (модель $M T_{2}$ ) уменьшает это расхождение для $\hbar \omega_{L}(X)$ до $1.5 \%$ и для $\hbar \omega_{T}(X)$ до $5.9 \%$, что близко к экспериментальной погрешности $5 \%$.

Представленные в [4] экспериментальные значения фононных частот для $\mathrm{Ar}$ при $p=20 \mathrm{GPa}$ имеют настолько большую погрешность, что делают сравнение с теоретическими расчетами бессмысленными. Другие измерения $\hbar \omega_{\lambda \mathbf{k}}$ при больших давлениях для КИГ нам не известны. Именно отсутствие качественного эксперимента делает весьма проблематичным тестирование теории по адекватному описанию $\hbar \omega_{\lambda \mathbf{k}}$ на границе зоны Бриллюэна, хотя область коротковолновых фононов является наиболее чувствительной к деталям теории даже при нулевом давлении [34].

В работе [26] представлены фононные дисперсионные кривые в симметричных направлениях волнового вектора $\mathbf{k}$ для всех кристаллов ряда $\mathrm{Ne}-\mathrm{Xe}$ при сжатии $\Delta V / V=0.7$, рассчитанные с использованием экспериментальных значений упругих модулей в разработанной авторами динамической модели. По мнению авторов [26], результаты расчетов совпадают с нашими (вероятно, имеются ввиду расчеты $\hbar \omega_{\lambda \mathbf{k}}$ в модели $M T_{0}$ ).

В работе [45] авторы представили $a b$ initio исследование динамики ГЦК-Хе на основе DFT. Найдено, что для Хе в ГЦК-фазе все фононные моды монотонно растут с давлением до $100 \mathrm{GPa}$, выше которого поперечные акустические моды в точках $X$ и $L$ начинают „размягчаться“. Как видно из рис. $1, d, 2, c$ и таблицы в Хе „размягчаются“поперечные моды в точке $L$ и в направлении $\Sigma$ (мода $T_{1}$ ), причем, при несколько меньшем давлении $\left(p_{c}=88 \mathrm{GPa}\right)$.

Для всех КИГ наблюдается такое же несоответствие $p_{c}$, рассчитанных нами (см. таблицу) и рассчитанных в DFT $[10,13]$. Аргументом в пользу наших результатов является экспериментально полученные для Хе давление ГЦК-ГПУ-перехода равные $75 \mathrm{GPa}$ [42] и металлизация при 128-132 GPa [15].

На наш взгляд, завышенные $p_{c}$, полученные в DFT могут означать некорректный учет деформации электронных оболочек. Модель Толпыго позволяет явно учесть эту деформацию в парном и трехчастичном приближении, что и приводит к размягчению „критических“ колебаний и абсолютной неустойчивости ГЦК-кристаллов инертных газов при высоких давлениях $\left(p>p_{c}\right)$.

В заключение, отметим аналогию $\mathrm{c}$ абсолютной неустойчивостью ОЦК-решетки щелочных металлов. Как показано в [46] (с. 286-288, рис. 4, 17), в ОЦК-кристаллах $\mathrm{K}, \mathrm{Rb}, \mathrm{Cs}$ при $p>p_{\text {c }}$ для сжатий $u=\Delta V / V_{0}$ равных $0.62,0.56$ и 0.52 соответственно, наблюдается обращение в нуль частоты „критической“ ветви $\Sigma_{4}$ в точке $\mathbf{k}[0.4 ; 0.4 ; 0]$, т.е. наступает абсолютная неустойчивость указывающая на необходимость фазового перехода.

\section{Список литературы}

[1] R.J. Hemley, H.K. Ashcroft. Phys. Today 51, 26 (1998).

[2] H. Shimizu, N. Saitoh, S. Sasaki. Phys. Rev. B 57, 230 (1998).

[3] M. Krish. J. Raman Spectrosc. 34, 628 (2003).

[4] F. Occelli, M. Krisch, P. Loubeyre, F. Sette, R. Le Toullec, C. Masciovecchio, J-P. Rueff. Phys. Rev. B 63, 224306 (2001).

[5] D. Young. Phase Diagrams of Elements. University of California Press, Berkeley (1991).

[6] N.H. March. In: Advances in high pressure research. / Ed. R.S.Bradley. Academic Press, N.Y. (1969). V. 3 P. 241.

[7] Y. He, X. Tang, Y. Pu. Physica B 405, 4335 (2010).

[8] L.W. Finger, R.M. Hazen, G. Zou, H.K. Mao, P.M. Bell. Appl. Phys. Lett. 39, 892 (1981).

[9] M. Ross, H.K. Mao, P.M. Bell, J.A. Xu. J. Chem. Phys. 85, 1028 (1986).

[10] I. Kwon, L.A. Collins, J.D. Kress, N. Troullier. Phys. Rev. B 52, 21, 15165 (1995).

[11] И.В. Александров, А.Н. Зисман, С.М. Стишов. ЖЭТФ 65, 371 (1987).

[12] A. Polian, J.M. Besson, M. Grimsditch, W.A. Grosshans. Phys. Rev. B 39, 1332 (1989).

[13] J. Hama, K. Suito. Phys. Lett. A 140, 117 (1989).

[14] H. Cynn, C.S. Yoo, B. Baer, V. Iota-Herbei, A.K. McMahan, M. Nicol, S. Carlson. Phys. Rev. Lett. 86, 4552 (2001).

[15] K.A. Goettel, J.H. Eggert, I.F. Silvera, W.C. Moss. Phys. Rev. Lett. 62, 665 (1989).

[16] D. Errandonea, B. Schwager, R. Boehler, M. Ross. Phys. Rev. B 65, 214110 (2002).

[17] D. Errandonea, R. Boehler, S. Japel, M. Mezouar, L.R. Benedetti. Phys. Rev. B 73, 092106 (2006).

[18] S. Sasaki, N. Wada, T. Kumi, H. Shimizu. J. Raman Spectroscopy. 40, 121 (2009).

[19] N.V. Krainyukova, R.E. Boltnev, E.P. Bernard, V.V. Khmelenko, D.M. Lee, V. Kiryukhin. Phys. Rev. Lett. 109, 245505 (2012).

[20] K. Takemura, T. Watanuki, K. Ohwada, A. Machida, A. Ohmura, K. Aoki. J. Phys.: Conf. Series 215, 012017 (2010). 
[21] Ю.Э. Китаев, А.Г. Панфилов, Е.S. Tasci, M.I. Aroyo. ФТТ 57, 2228 (2015).

[22] R.A. Evarestov, Yu.E. Kitaev. J. Appl. Cryst. 49, 1572. (2016).

[23] Yu.A. Freiman, S.M. Tretyak. Low Temp. Phys. 33, 545 (2007).

[24] A. Grechnev, S.M. Tretyak, Yu.A. Freiman. Low Temp. Phys. 36, 333 (2010).

[25] N.V. Krainyukova. Low Temp. Phys. 37, 435 (2011).

[26] И.О. Мачихина, В.Е. Холодовский. Изв. Самарск. науч. центра РАН 14, 116 (2012).

[27] В.Ю. Бодряков. ЖТФ 83, 101 (2013).

[28] L. Shulenburger, M.P. Desjarlais, T.R. Mattsson. Phys. Rev. B 90, 140104 (2014).

[29] C. Tian, F. Liu, L. Cai, H. Yuan, H. Chen, M. Zhong. J. Chem. Phys. 143, 174506 (2015).

[30] P. Schwerdtfeger, R. Tonner, G.E. Moyano, E. Pahl. Angew. Chem. Int. Ed. 55, 12200 (2016).

[31] M.G. Medvedev, I.S. Bushmarinov, J. Sun, J.P. Perdew, K.A. Lyssenko. Science 355, 49 (2017).

[32] P. Schwerdtfeger, K.G. Steenbergen, E. Pahl. Phys. Rev. B 95, 214116 (2017).

[33] Е.П. Троицкая, Е.Е. Горбенко, Е.А. Пилипенко. ФНТ 42 , 526 (2016).

[34] Е.П. Троицкая, Вал.В. Чабаненко, Е.Е. Горбенко, Е.А. Пилипенко. ФТТ 57, 114 (2015).

[35] Е.А. Пилипенко, Е.П. Троицкая, Е.Е. Горбенко. ФТТ 60, 151 (2018).

[36] М. Борн, Хуан Кунь. Динамическая теория кристаллических решеток. ИЛ, М. (1958). 488 с.

[37] Е.П. Троицкая, Вал.В. Чабаненко, И.В. Жихарев, Е.Е. Горбенко. ФТТ 53, 1555 (2011).

[38] F. Clementi, C. Roetti. Roothan-Hartree-Fock atomic wave functions. At. Data Nucl. Data Table 14, 3-4 (1974).

[39] Е.П. Троицкая, В.В. Чабаненко, Е.Е. Горбенко. Физика и техника высоких давлений 16, 25 (2006).

[40] Л.Д. Ландау, Е.М. Лифшиц. Статическая физика. Наука, М. (1976). Ч. 1. $584 \mathrm{c}$.

[41] Е.Е. Горбенко, Е.П. Троицкая, Е.А. Пилипенко. ФТТ 59, 126 (2017).

[42] A.P. Jephcoat, H.K. Mao, L.W. Finger, D.F. Lox, R.J. Hemley, C.S. Zha. Phys. Rev. Lett. 59, 2, 2670 (1987).

[43] E. Pechenic. I. Kelson, G. Makov. Phys. Rev. B 78, 134109 (2008).

[44] Е.П. Троицкая, В.В. Чабаненко, Е.Е. Горбенко. ФТТ 49, 2055 (2007).

[45] J.K. Dewhurst, R. Ahuja, S. Li, B. Johansson. Phys. Rev. Lett. 88, 7, 075504 (2002).

[46] V.G. Bar'akhtar, E.V. Zarochentsev, E.P. Troitskaya. Theory of adiabatic potential and atomic properties of simple metals. Gordon\&Breach, London (1999). 317 p.

Редактор Т.Н. Василевская 